\title{
Incidência de Neoplasias Malignas de esôfago na população de Volta Redonda, Rio de Janeiro
}

\author{
Esophagus malignant neoplasms incidence in the population of Volta \\ Redonda, Rio de Janeiro
}

\author{
Walter Manuel Tavares Nóbrega Junior ${ }^{1}$ \\ Nathalia Monerat Pinto Blazuti Barreto ${ }^{1}$ \\ Silvio Delfini Guerra' \\ Marcus Vinicius Ribeiro Carvalho² \\ Loreley Andrade Luderer ${ }^{3}$ \\ Juliana de Paula Machado ${ }^{4}$ \\ Sergio Elias Vieira Cury ${ }^{5}$
}

\footnotetext{
1 Discente do Curso de Medicina do Centro Universitário de Volta Redonda - UniFOA.

2 Discente do Curso de Odontologia do Centro Universitário de Volta Redonda - UniFOA.

3 Docente do Curso de Odontologia do Centro Universitário de Volta Redonda - UniFOA.

4 Médica do Programa de Residência em Pediatria do Hospital Universitário Sul Fluminense

5 Docente dos Cursos de Medicina e Odontologia do Centro Universitário de Volta Redonda - UniFOA.
}

\section{RESUMO}

Objetivo: Avaliar a incidência de neoplasias malignas do esôfago na população de Volta Redonda, por meio de um estudo retrospectivo, em 10 anos, nos registros de diagnósticos histopatológicos. Método: foram obtidos 10.000 registros, pertencentes ao acervo da disciplina de Patologia Geral do Curso de Odontologia do UniFOA, oriundos do extinto Hospital da Companhia Siderúrgica Nacional, na cidade de Volta Redonda, Rio de Janeiro, Brasil, registrados no período compreendido entre os anos de 1990 a 2000. Foram selecionados os casos diagnosticados como de neoplasias malignas que apresentaram o esôfago como sítio primário. Neoplasias metastáticas não foram consideradas. Resultados: Foram encontrados 1280 casos diagnosticados como de neoplasias malignas no referido serviço. Dentre esses, $3,90 \%(n=50)$ apresentaram como sitio primário o esôfago. A idade variou de 42 a 76 anos, com média de 65,6 anos, com predominância na faixa etária entre 60 e 69 anos $(n=29)$. Ocorreram 38 casos no gênero masculino (76\%) e 12 no feminino (24\%). Em relação ao diagnostico histopatológico, a maioria dos casos foi de carcinomas epidermóides ( $\mathrm{n}=35,70 \%)$.

\section{PALAVRAS-CHAVE}

Neoplasia maligna; trato digestivo; sitio primário.

\begin{abstract}
Objetive: The aim of this study is To evaluate the incidence of esophagus malignant neoplasms in the population of Volta Redonda, through a retrospective study in 10 years on the records of histopathological diagnoses. Methods: Ten thousand records were obtained, belonging to the collection of the discipline of General Pathology, UniFOA Dental School coming of the former Hospital of the National Steel Company in the city of Volta Redonda, Rio de Janeiro, Brazil, recorded between 1990 to 2000. esophagus as the primary site. Metastatic tumors were not considered. Results: Were found 1280 cases diagnosed as malignant neoplasms of the related service. Of these, $3.90 \%(n=50)$ had as primary site of the esophagus. The age ranged from 42 to 76 years, mean 65.6 years, predominantly in the age group between 60 and 69 years $(n=29)$. Thirty eight cases occurred in males (76\%) and 12 females (24\%). Regarding the histopathological diagnosis, most of cases were squamous cell carcinomas $(n=35,70 \%)$.
\end{abstract}

\section{Keywords}

Malignancy; digestive tract; primary site.

\section{Como você deve citar?}

CURY, Sergio Elias Vieira. Incidência de Neoplasias Malignas de esôfago na população de Volta Redonda, Rio de Janeiro. Cadernos UniFOA, Volta Redonda, n. 27, p. 89-94, abr. 2015. 


\section{INTRODUÇÃO}

A prevalência de neoplasias, sejam elas benignas ou malignas, tem gerado estudos em vários países, no intuito principal de mapear a qualidade de vida dos pacientes, em especial no que tange aos diversos tipos e diversos tecidos envolvidos.

Neoplasias têm sido relatadas como "proliferações locais de clones celulares, cuja reprodução foge ao controle normal, e que tendem para um tipo de crescimento autônomo e progressivo, e para a perda de diferenciação." Classicamente, têm sido classificadas em benignas e malignas, de acordo com seu grau de diferenciação celular, capacidade de invadir tecidos adjacentes ao local de origem e possibilidade de levar o paciente ao óbito (NEVILLE et al. 2010; MITCHEL et al., 2012).

O conhecimento dos principais tipos de neoplasias numa população traz luz ao conhecimento, contribuindo a escolha e adoção de estratégias de saúde, principalmente, em relação aos fatores de risco que, por ventura, possam estar associados a elas.

Objetivo do presente artigo é avaliar a incidência de neoplasias malignas do trato digestivo na população de Volta Redonda, por meio de um estudo retrospectivo de 10 anos, nos registros de diagnósticos histopatológicos, do arquivo da disciplina de Patologia geral do Curso de Odontologia do UniFOA.

\section{REVISÃO DA LITERATURA}

O termo "neoplasia maligna" foi definido por Willis, em 1952, em seu livro intitulado "A propagação de tumores no corpo humano", como sendo uma massa anormal de tecido, cujo crescimento excede aquele dos tecidos normais, e não está coordenado com ele, persistindo da mesma maneira excessiva após o término do estímulo que induziu a alteração". Mitchell et al. (2012) acrescentou a essa definição, que a massa anormal carece de propósito e é predadora do hospedeiro, sendo praticamente autônoma.

O câncer de esôfago é uma neoplasia maligna relativamente incomum e extremamente letal. Acomete mais homens do que mulheres, surgindo com maior frequência depois dos 50 anos de idade (maior incidência aos 65) e parece estar associado a níveis socioeconômicos mais baixos (ZAN et al., 2005; QUEIROGA; PERNAMBUCO, 2006). Segundo o Instituto Nacional do Câncer (INCA - Brasil 2012), o câncer de esôfago é o sexto mais comum entre os homens e o décimo quinto entre as mulheres. No mundo, ocupa a sexta posição, mas é o principal tipo de câncer nos países da Ásia central (IGISSINOV et al., 2012).

Histologicamente, o CAE pode ser dividido em dois tipos: carcinoma epidermóide ou escamoso e adenocarcinoma. 0 primeiro é o mais frequente e é derivado do epitélio pavimentoso estratificado presente na mucosa esofageana, correspondendo em média a $85 \%$ dos casos, acometendo principalmente os terços médio e inferior do esôfago. 0 segundo ocorre na parte distal do órgão, desenvolve-se no interior do epitélio colunar displásico, principalmente na junção esôfago-gástrica/cárdiaque. Por ter íntima relação com a doença do refluxo gastroesofágico (DRGE) e com a metaplasia de Barret, vem ganhando espaço na literatura nos últimos anos (ZAN et al., 2005; QUEIROGA; PERNAMBUCO, 2006; NEMETH et al., 2012; MITCHELL et al., 2012).

Dentre os fatores de risco para o câncer de esôfago estão o tabagismo, etilismo, exposição à radiação, acalasia, megaesfôfago chagásico. Outros fatores alimentares são as carências nutricionais de vitaminas A e C, de ferro e zinco (TERCIOTTI Jr et al., 2011), a alta ingestão de gordura e a pouca de carboidratos. (LAGERGREN; MATTSSON; LAGERGREN, 2013). Fatores regionais como a ingestão de 
Walter Manuel Tavares Nóbrega Junior I Nathalia Monerat Pinto Blazuti Barreto / Silvio Delfini Guerra Marcus Vinicius Ribeiro Carvalho / Loreley Andrade Luderer I Juliana de Paula Machado / Sergio Elias Vieira Cury

mate, bebidas quentes e churrasco, comuns na região Sul do Brasil também estão associados (GUERRA; GALLO; SILVA MENDONÇA, 2005). Também podem contribuir com o surgimento do câncer de esôfago a síndrome de Plummer-Vinson, a Tilose palmar e o Papiloma Vírus Humano (HPV) (TERCIOTTI Jr et al., 2011), além da DRGE e da metaplasia de Barret já citadas.

Sintomas como diminuição do apetite, náusea, vômito, emagrecimento e odinofagia podem estar presentes, mas o principal, a disfagia, ocorre de maneira tardia, dificultando o diagnóstico precoce, acarretando em maior mortalidade desse câncer (MONTEIRO et al., 2009), de modo que a taxa de mortalidade se aproxima da taxa de novos casos (MELLO et al., 2010). A sobrevida do paciente tem íntima relação com o estágio da doença, apresentando valores que variam desde $75 \%$ em cinco anos (naqueles em estádio 0 ) a menores que $5 \%$, quando o estádio é IV.

\section{METODOLOGIA}

Por meio de uma análise retrospectiva, foram obtidos 10.000 registros de diagnósticos histopatológicos, pertencentes ao acervo da disciplina de Patologia Geral do Curso de Odontologia do UniFOA, oriundos do extinto Hospital da Companhia Siderúrgica Nacional na cidade de Volta Redonda, Rio de Janeiro, Brasil, registrados no período compreendido entre os anos de 1990 a 2000.

Inicialmente, foram selecionados os casos diagnosticados como de neoplasias malignas e, posteriormente, dentre esses, separados os casos relatados como tendo o esôfago, o intestino e o reto como sítio primário. Neoplasias metastáticas não foram consideradas.

Obtidos os casos envolvendo cada órgão, foram selecionadas as lâminas com as secções teciduais coradas pela técnica da hematoxilina-eosina, para confirmação do diagnóstico, confirmação essa realizada por dois professores da disciplina.

Após a confirmação diagnóstica, os dados foram agrupados separadamente por ano do diagnóstico e tipo histológico do tumor, levando-se ainda em conta sexo e idade do paciente. Para organização dos dados foi utilizado o Microsoft Excel versão 2010.

Para avaliação dos critérios éticos, um Protocolo foi encaminhado ao Comitê de Ética em Pesquisa com Seres Humanos do Centro Universitário de Volta Redonda, que emitiu parecer favorável à sua realização e com registro na Comissão Nacional de Ética em Pesquisa do Ministério da Saúde (CAAE - 01234712.1.0000.5237, Parecer nº 23009 de 08/05/2012).

\section{RESULTADOS}

Foram encontrados 1.280 casos diagnosticados como de neoplasias malignas no referido serviço. Dentre esses, 3,90\% ( $n=50)$ apresentaram como sitio primário o esôfago.

A idade variou de 42 a 76 anos, com média de 65,6 anos, com predominância na faixa etária entre 60 e 69 anos (n=29) (Tabela 1). 
Tabela 1 - Idade

\begin{tabular}{ccc}
\hline IDADE & $\mathbf{n}$ & $\%$ \\
\hline 40 a 49 & 1 & 2 \\
\hline 50 a 59 & 6 & 12 \\
\hline 60 a 69 & 29 & 58 \\
\hline 70 a 79 & 14 & 28 \\
\hline TOTAL & $\mathbf{5 0}$ & 100 \\
\hline & Fonte: dos autores.
\end{tabular}

Ocorreram 38 casos no gênero masculino ( $76 \%$ ) e 12 no feminino (24\%), numa relação $3,16: 1$ (Tabela 2).

Tabela 2 - Gênero

\begin{tabular}{ccc}
\hline & $\mathbf{n}$ & $\%$ \\
\hline Masculino & 38 & 76 \\
\hline Feminino & 12 & 24 \\
\hline TOTAL & $\mathbf{5 0}$ & $\mathbf{1 0 0}$ \\
\hline & Fonte: dos autores.
\end{tabular}

Em relação ao diagnostico histopatológico, a maioria dos casos foi de carcinomas epidermóides $(n=35,70 \%)$, tendo sido encontrados 15 casos (30\%) de adenocarcinomas (Tabela 3 ).

Tabela 3 - Diagnóstico histopatológico

\begin{tabular}{ccc}
\hline & $\mathbf{n}$ & $\%$ \\
\hline Carcinoma Epidermóides & 35 & 70 \\
\hline Adenocarcinomas & 15 & 30 \\
\hline TOTAL & $\mathbf{5 0}$ & $\mathbf{1 0 0}$ \\
\hline & Fonte: dos autores.
\end{tabular}

\section{DISCUSSÃO}

A preocupação pela incidência do câncer de esôfago na população de Volta Redonda é reafirmada pelos dados do INCA, demonstrando que o mesmo é o sexto tipo de câncer mais incidente entre os homens e o décimo quinto entre as mulheres. 0 índice de 3,90\% encontrado não pode ser comparado a outro, por tratar-se de um levantamento em acervo específico, no qual a população era composta por indivíduos submetidos a biópsias num hospital de referência. Outros casos podem ter sido diagnosticados em outros serviços da cidade, o que impediu o cálculo para comparação, ficando o dado disponível para comparação com estudos futuros.

Nosso resultado demonstrou que o tumor acometeu 38 indivíduos do gênero masculino (76\%), estando de acordo com os achados de Zan et al. (2005) e Queiroga e Pernambuco (2006). Esses estudos relataram que o tumor surge com maior frequência depois dos 50 anos de idade, com maior incidência 
aos 65 anos; resultado semelhante ao encontrado em nosso estudo, no qual somente um indivíduo apresentou idade inferior a 50 anos, e a faixa etária de maior incidência ficou compreendida entre 60 e 69 anos. No estudo de Queiroga e Pernambuco (2006), ainda há relato de que a lesão parece estar associada a níveis socioeconômicos mais baixos, dado esse, porém, que não pôde ser avaliado em nosso estudo, por não constar nos registros do acervo utilizado.

Dentro da subdivisão dada pela característica histopatológica do tumor, a maioria encontrada em nosso estudo foi de carcinomas epidermóides ( $n=35,70 \%$ ), estando de acordo com as publicações de Zan et al. (2005), Queiroga e Pernambuco (2006) e Mitchell et al. (2012) . 0 índice encontrado (70\%) está abaixo dos $85 \%$ relatados pelos trabalhos citados, diferença que pode ser explicada pela diferença de raças e hábitos alimentares existentes entre a América do Sul e continentes como a América do Norte e a Europa, por exemplo.

\section{CONCLUSÃO}

A incidência encontrada foi de 3,9\%, com maior ocorrência na faixa etária entre 60 e 69 anos e no gênero masculino. 0 principal tipo histológico foi o Carcinoma epidermóide. Os dados obtidos são compatíveis com os relatados na literatura. 


\section{REFERÊNCIAS}

BRASIL. Instituto Nacional do Câncer. Estimativa 2012 Incidência do Câncer no Brasil. Disponível em: <http://www.inca.gov.br/estimativa/2012/estimativa20122111.pdf>. Acesso em: 10/04/2012.

GUERRA, M.G.; GALLO, C.V.M.; SILVA MENDONÇA G.A. Risco de câncer no Brasil: tendências e estudos epidemiológicos mais recentes. Rev Bras Cancerol v. 51, n. 3, p. 227-34, 2005.

IGISSINOV, S.; IGISSINOV, N.; MOORE, M.A. et al. Epidemiology of Esophageal Cancer in Kazakhstan. Asian Pacific J Cancer Prev v. 13, p. 833-6, 2012.

LAGERGREN, K.; MATTSSON, F.; LAGERGREN J. Abdominal fat and male excess of esophageal adenocarcinoma. Epidemiology v. 24, n. 3, p. 465-6, 2013.

MELLO, B.S.; LUCENA, A.F.; ECHER, I.C. et al. Pacientes com câncer gástrico submetidos à gastrectomia: uma revisão integrativa. Rev Gaúcha Enferm v. 31, n. 4, p. 803-11, 2010.

MITCHELL, C.; ABBAS, A.K.; KUMAR, V.Y.; FAUSTO, N.; ASTER. J.Ç. Robins \& Cotran Patologia - Bases Patológicas das doenças. 9 ed. São Paulo: Elsevier, 2012.

MONTEIRO, L.M.N.; ARAÚJO, D.F.; BASSETTI-SOARES, E. et al. Câncer de Esôfago: Perfil das manifestações clínicas, histologia, localização e comportamento metastático. Rev Bras Cancerol v. 55, n. 1, p. 27-32, 2009.

NEMETH, I.B.; ROSZTOCZY, A.; IZBEKI, F. et al. A renewed insight into Barrett's esophagus:comparative histopathological analysis of esophageal columnarmetaplasia. Dis Esophagus v. 25, n. 5, p. 395-402, 2012.

NEVILLE, B. W.; DAMM, D. D.; ALLEN, C. M. et al. Patologia oral \& maxillofacial. $3^{a}$ ed. Rio de Janeiro: Elservier; 2010.

QUEIROGA, R.C.; PERNAMBUCO, A.P. Esophageal Cancer: Epidemiology, Diagnosis, and Treatment. Rev Bras Cancerol v. 52, n. 2, p. 173-8, 2006.

TERCIOTI JUNIOR, V.; LOPES, L.R.; COELHO NETO, J.S. et al. Eficácia local e complicações da terapêutica neoadjuvante no carcinoma epidermóide do esôfago: radioterapia versus radioterapia associada à quimioterapia. Rev Col Bras Cir v. 38, n. 4; p. 227-31, 2011.

WILLIS, R. The Spread of Tumors in the Human Body. Lodon: Butterworth \& Co, 1952.

ZAN, T.A.B.; FRANÇA, F.C.; MUNIZ, M.P. et al. Prevalence of lung abnormalities in 55 patients with esophageal ccncer. Radiol Bras [online]. v. 38, n. 1, p. 11-5, 2005. 